\title{
Theory and applications of the Lugiato-Lefever Equation ${ }^{\star}$
}

\author{
Yanne K. Chembo ${ }^{1, a}$, Damià Gomila ${ }^{2}$, Mustapha Tlidi ${ }^{3}$, and Curtis R. Menyuk ${ }^{4}$ \\ ${ }^{1}$ GeorgiaTech-CNRS Joint International Laboratory, Atlanta, USA \\ ${ }^{2}$ Instituto de Física Interdisciplinar y Sistemas Complejos (IFISC), Palma de Mallorca, Spain \\ ${ }^{3}$ Université Libre de Bruxelles, Brussels, Belgium \\ ${ }^{4}$ University of Maryland Baltimore County, Baltimore, USA
}

Received 4 September 2017

Published online 23 November 2017 - (c) EDP Sciences, Società Italiana di Fisica, Springer-Verlag 2017

The Lugiato-Lefever Equation was first introduced in 1987 by Luigi Lugiato and René Lefever in Phys. Rev. Lett. 58, 2209 (1987). The purpose of their article was to analyze spatiotemporal pattern formation in a dissipative, diffractive and nonlinear optical cavity submitted to a continuous laser pump. In order to achieve that goal, they had derived a model - later referred to as the LLE - that was a damped, driven and detuned version of the wellknown nonlinear Schrödinger equation that is commonly used in the area of nonlinear fiber optics. The LLE rapidly arose as a paradigm for the understanding of complex phenomena in dissipative and nonlinear optical cavities. This model has also been successfully applied by Haelterman, Trillo, and Wabnitz to the case of dispersive optical ring cavities [Opt. Commun. 91, 410 (1992)]. More recently, it has also become the basic model for the study of broadband microresonator-based optical frequency combs [Opt. Lett. 26, 2845 (2011)], which were experimentally evidenced for the first time in Nature 450, 1214 (2007). Thus, in the 30 years since it was first proposed, the LLE has become a widely used model to investigate the dynamical properties of laser fields confined in nonlinear optical resonators.

From a fundamental viewpoint, the LLE has led to the prediction and analysis of various intra-cavity structures such as solitons (bright and dark; spatial and temporal; breathers), Turing patterns (rolls, hexagons), and even spatiotemporal chaos. With respect to applications, this equation has also been found to be the best framework for the theoretical investigation of Kerr optical frequency comb generation using whispering gallery mode cavities or integrated ring resonators. It should be noted that $T$. Hänsch and J. Hall obtained the

\footnotetext{
* Contribution to the Topical Issue "Theory and Applications of the Lugiato-Lefever Equation", edited by Yanne K. Chembo, Damia Gomila, Mustapha Tlidi, Curtis R. Menyuk.

${ }^{a}$ e-mail: yanne.chembo@femto-st.fr
}

Nobel Prize of Physics in 2005 for their pioneering work on optical frequency comb technology, and the most recent versions of these combs are theoretically described by the LLE with unprecedented precision (dynamic range higher than $100 \mathrm{~dB}$, and frequency span larger than one octave). These Kerr combs are expected to revolutionize the generation of ultra-stable lightwave and microwave signals for aerospace engineering, optical communication networks, and microwave photonic systems.

An additional example of the far-reaching importance of the LLE is the quantum version of this equation, proposed in 1992 by Lugiato and Castelli, forecasting the possibility of squeezing [Phys. Rev. Lett. 68, 3284 (1992)]. This pioneering theoretical research was recently confirmed experimentally in a work showing that Kerr combs can generate squeezed states of light, thereby opening the way to on-chip optical squeezing for quantum engineering/communication applications [Phys. Rev. Appl. 3, 044005 (2015)].

This topical issue, which celebrates the 30 th anniversary of the LLE, is intended to provide an overview of current research in relation to the theory and applications of that equation. It features an introductory article co-authored by Luigi Lugiato, and contains 15 contributed articles in total. We are very pleased to provide a diverse overview of the current research associated with the LLE, which includes spatial dissipative structures in optical cavities, temporal dissipative structures in fiber ring resonators, the mathematical analysis of the LLE, and of course, Kerr optical frequency combs in whispering gallery mode resonators.

We would like to thank the authors for their valuable contributions to this topical issue. We modestly hope as well that their articles will provide a convincing display of the multidisciplinary and contemporary nature of the LLE, which can be expected, even in the long term, to strengthen our understanding of light-matter interaction at the semi-classical and quantum levels. 